\section{$\underset{\substack{\text { hommes } \\ \text { \& migrations }}}{ }$}

\section{Hommes \& migrations}

Revue française de référence sur les dynamiques

migratoires

\section{$1297 \mid 2012$}

Migrations en création

\title{
Staff Benda Bilili
}

Bouger le monde !

\section{François Bensignor}

\section{Q OpenEdition \\ 1 Journals}

\section{Édition électronique}

URL : http://journals.openedition.org/hommesmigrations/1332

DOI : 10.4000/hommesmigrations. 1332

ISSN : 2262-3353

Éditeur

Musée national de l'histoire de l'immigration

\section{Édition imprimée}

Date de publication : 1 mai 2012

Pagination : 161-166

ISSN : 1142-852X

\section{Référence électronique}

François Bensignor, «Staff Benda Bilili », Hommes \& migrations [En ligne], 1297 | 2012, mis en ligne le 29 mai 2013, consulté le 24 septembre 2020. URL : http://journals.openedition.org/ hommesmigrations/1332; DOI : https://doi.org/10.4000/hommesmigrations.1332

Ce document a été généré automatiquement le 24 septembre 2020.

Tous droits réservés 


\title{
Staff Benda Bilili
}

\author{
Bouger le monde!
}

François Bensignor

Après un premier album, Très Très Fort, plébiscité par le public européen et couronné par le Womex Award 2009, après le film Benda Bilili ! présenté au Festival de Cannes 2010 en ouverture de la Quinzaine des réalisateurs, après quatre tournées internationales, où il ne manque jamais de faire danser les foules, Staff Benda Bilili présente son deuxième album, Bouger le monde!, en septembre 2012. Une belle occasion de retracer l'épopée de ce groupe de handicapés de Kinshasa en compagnie de Ricky, fondateur et maître à penser de ce combo, dont l'histoire exemplaire peut être méditée en ces temps de crise. Le groupe congolais Staff Benda Bilili est né en 2003 dans les rues de Kinshasa de la volonté d'un petit noyau d'amis handicapés : Ricky, Coco, Théo et Djuana. Autour de ces passionnés de musique se sont greffés deux autres musiciens handicapés, Zadis et Kabamba. Mais aussi deux jeunes gens valides, Cavalier et Cubain, qui assurent la partie rythmique basse, batterie. Ils sont rejoints enfin par deux shégés, comme on nomme les enfants des rues de la capitale congolaise: Randy, le plus jeune du groupe, et Roger, extraordinaire virtuose d'un petit instrument à corde qu'il s'est inventé avec des matériaux de récupération. Personne pourtant n'aurait jamais entendu parler de cet ensemble improbable sans sa rencontre avec deux cinéastes français, Renaud Barret et Florent de La Tullaye. Partis en 2004 à Kinshasa, ils réalisent, en immersion totale avec les jeunes musiciens du ghetto, le documentaire Jupiter's Dance, axé sur le chanteur Jupiter Bokondji et son groupe Okwess International. Par l'intermédiaire de Cubain, jeune batteur qui travaille avec plusieurs groupes, ils font la connaissance de cet étrange combo de musiciens handicapés, qui circulent sur d'incroyables tricycles, poussés par des shégés dans les rues défoncées de la capitale congolaise. En 2004, en 2006, au gré de leurs allers-retours successifs, les deux Français font des images avec le groupe, tout en envisageant d'enregistrer son premier album. Ricky, doyen, fondateur et tête pensante du Staff Benda Bilili, raconte: "Quand Florent et Renaud sont venus me rencontrer, ils m'ont dit qu'ils voulaient faire un documentaire sur les enfants des rues. Avant d'enregistrer notre musique, ils voulaient nous filmer, parce que, avec ce groupe, tout ce que l'on faisait se passait dans la rue: manger, préparer nos instruments, tout." Une véritable complicité s'installe progressivement entre l'équipe de musiciens et les deux cinéastes 
qui n'hésitent pas à partager leurs conditions de vie. Ils ont appris le lingala afin d'être en mesure de les comprendre et de se faire comprendre. À la fin de 2006, ils trouvent en la personne de Vincent Kenis le réalisateur idéal pour le disque du Staff. Producteur de la série Congotronics pour le label Crammed Discs, ce Belge mordu de Kinshasa est parmi les meilleurs connaisseurs des musiques congolaises. Il a réalisé certaines compilations incontournables de rumba des années cinquante, comme les premiers enregistrements de Franco. On lui doit également les disques de formidables groupes tradi-modernes, dont Swede Swede, Konono n ${ }^{\circ} 1$ ou Kassaï All Stars. Il va enregistrer le Staff en plein air, dans l'endroit même où les musiciens ont l'habitude de répéter : le Parc zoologique de Kinshasa. Le film Benda Bilili! (Au-delà des apparences) témoigne magnifiquement de l'aventure. Grande leçon d'optimisme, il accompagne le parcours improbable du groupe depuis la fange des rues de Kinshasa jusqu'à ses tout premiers succès de 2009 sur les scènes européennes. "Pendant notre première tournée, on avait le trac. Entrer sur scène devant 2000 personnes, ça ne nous était jamais arrivé. Et puis, on s'est habitué", dit Ricky, fataliste... La joie, la détermination, l'amour de la musique semblent alimenter l'incroyable énergie du Staff, au mépris des embûches. Tournant dramatique de l'histoire bien réelle de ces musiciens, alors que la première partie du rêve commence à voir le jour avec l'enregistrement de leurs premières chansons, un incendie ravage le Centre pour handicapés dans lequel vivent plusieurs d'entre eux avec leur famille. " Après cet incendie, le ministère des Affaires sociales nous a déplacés vers un autre centre”, se souvient Ricky. La suite de l'aventure va transcender ce moment noir où tout faillit capoter. Florent raconte: "Nous sommes revenus en 2007 avec un peu plus de moyens, de façon à prendre en charge les musiciens le temps des sessions. Car leur vie dans la rue les bouffe littéralement." La consécration de 2010, lorsque le film est présenté en ouverture de la Quinzaine des réalisateurs au Festival de Cannes, n'en sera que plus belle. C'est la reconnaissance du combat incessant et du travail formidable qu'il a fallu déployer pour parvenir à hisser le Staff Benda Bilili où il est aujourd'hui, à faire danser des foules en délire sur les scènes du monde.

\section{Ricky \& Roger}

2 Né à Kinshasa en 1955, Ricky, de son vrai nom Léon Likabu, est issu de l'ethnie Mungelema du territoire de Banalia, dans la région de Kisangani. Son père, originaire de ce chef-lieu de la province orientale très en amont du fleuve Congo, est venu travailler à Kinshasa, où sa famille s'est agrandie. Arrivée la retraite, doté de sa pension, il retourne à Kisangani, où son fils le suit, laissant sa mère dans la capitale. Mais à la mort du père, Ricky décide de redescendre le fleuve Congo pour retrouver sa mère à Kinshasa et s'installer auprès d'elle. Atteint de la polio enfant, Ricky n'a pu être soigné. Il en résulte un handicap permanent, comme c'est le cas pour quatre autres membres de son groupe. L'un se déplace avec des béquilles, quatre évoluent en chaise roulante. Trois jeunes gens valides les accompagnent à la batterie, à la basse et en solo au satonge. Ce monocorde unique est constitué d'un petit arc de bois courbe, planté dans une boîte de conserve, relié aux extrémités par un fil de fer. S'il s'avère particulièrement difficile à manier, son inventeur, Roger Landu, parvient à en tirer des sons proches de ceux d'une guitare électrique avec une dextérité, une justesse et une vélocité tout à fait prodigieuses. Aujourd'hui âgé de 21 ans, Roger est le plus jeune et certainement le plus talentueux musicalement des huit membres du Staff Benda Bilili. Petit garçon, il a vécu les dérives des shégés, communauté vouée à l'errance, à la drogue 
et à la violence. Recueilli à l'âge de 13 ans, il est depuis encadré par Ricky, le vieux sage. Roger est un personnage essentiel du film de Renaud Barret et Florent de La Tullaye. Ils avaient fait sa connaissance fugitive lors de leur premier séjour en 2004, dans le ghetto de Kinshasa, mais l'avaient aussitôt perdu de vue. L'année suivante, les deux Français retrouvent par hasard un Roger de 14 ans, qu'ils introduisent aussitôt auprès du Staff Benda Bilili. Les vieux handicapés vont devenir ses "papas et grands frères", selon les propres mots du garçon. Adolescent timide caché derrière des lunettes de soleil, on le voit progressivement s'épanouir dans le film. Mais aujourd'hui, c'est un jeune homme charmeur de 21 ans à la voix affirmée et au jeu tout en délicatesse, que l'on retrouve en scène lors de la quatrième tournée internationale du groupe. En quelques années, le virtuose du satonge s'est construit une personnalité musicale rayonnante. "Quand j'ai rencontré Ricky, il m'a dit qu'un jour je deviendrai le chef d'orchestre du Staff Benda Bilili. J'ai donc une grande responsabilité, il faut que je continue de travailler", disait-il en 2010. Dont acte!

\section{Une vocation ancienne}

3 En 1974, était organisé à Kinshasa l'évènement le plus emblématique du rapprochement entre le monde afro-américain, libéré de la ségrégation raciale instituée, et l'Afrique décolonisée, en pleine phase idéologique d'affirmation de ses racines culturelles africaines. Baptisé "Rumble in the Jungle" par son promoteur américain, le sulfureux Don King, le légendaire combat de boxe opposant le champion du monde Muhammad Ali (ex-Cassius Clay) à George Foreman se tenait dans le gigantesque stade de Kinshasa. Sans doute le plus médiatisé alors sur la planète, ce choc de titans est précédé par trois concerts extraordinaires proposant douze heures de musique. L'affiche de rêve présente un florilège de stars noires auréolées de succès internationaux : James Brown, B.B. King, Sister Sledge, Miriam Makeba, Celia Cruz, The Crusaders, Bill Withers, Big Black, The Spinners. Dans son film When We Where Kings, le réalisateur Leon Gast a immortalisé cet événement du point de vue de la boxe et surtout de Ali. Trente-cinq ans plus tard, son chef monteur Jeffrey Levy-Hinte replongera dans les dizaines d'heures de rushs filmés pour l'occasion et en tirera Soul Power, un film saisissant entièrement consacré à l'événement musical. Ricky était parmi la foule venue en masse applaudir ces héros de la musique noire. Pour lui, ce fut le déclencheur de sa passion pour la musique. Il raconte : "J'étais là dans le stade pour écouter James Brown et tous les autres! Et c'est à partir de là que je me suis mis à la musique. J'ai commencé à chanter et à fabriquer des guitares. Je suis allé chanter dans une chorale d'église. On me donnait chaque fois quelques bonbons ou des biscuits, mais je me suis dit que ce n'était pas comme ça que je gagnerais ma vie. Il fallait que j'aille faire de la musique ailleurs." C'est ainsi que Ricky compose et écrit ses premières chansons. Mais pour les musiciens handicapés à Kinshasa, il n'y a guère d'autre moyen de gagner un peu d'argent qu'en tendant le chapeau devant des lieux fréquentés par des riches et des étrangers. Or Ricky tient à préciser qu'il n'a jamais vécu de la mendicité : "Jeune, j'ai commencé par exercer le métier de couturier. J’ai toujours gagné ma vie. J'étais déjà tailleur quand je me suis marié." Il a maintenant deux épouses régulières, l'une handicapée, l'autre valide, dont il a eu cinq enfants. Avec ses vieux amis, qui sont les membres fondateurs du Staff Benda Bilili, Ricky a également vécu du commerce entre Kinshasa et Brazzaville. Une règle édictée par le président Mobutu voulait en effet que les handicapés soient exemptés de taxes douanières. Aussi 
servaient-ils d'intermédiaires entre les nombreux commerçants qui devaient faire transiter leurs marchandises entre les deux Congo.

\section{Le nouveau son du Congo}

4 Si la rumba congolaise, inventée dans les années cinquante, sert de modèle à tous les nouveaux courants qui se sont succédé à Kinshasa depuis, la perpétuant en la modernisant et en lui apportant de nombreuses variantes au long de plus de cinq décennies, Staff Benda Bilili ne s'inscrit pas vraiment dans cette transmission. Inspirée par les rythmiques binaires du funk et de la soul américaine, sa musique tranche avec le reste de la production locale. C'est peut-être d'ailleurs la clé de son succès auprès des Européens. Peu initiés aux rythmes des danses congolaises, qui peuvent atteindre un haut degré de sophistication dans la polyrythmie et la façon dont s'élaborent les architectures orchestrales, ces publics ont un accès plus direct à la pulsion instillée par l'orchestre des handicapés. Certains de ses morceaux puisent volontiers dans le balancement reggae, lui aussi familier à l'oreille occidentale. Incontestablement, Staff Benda Bilili a inventé un son original, en utilisant des instruments qui font sa marque et auxquels les musiciens restent très attachés, comme l'affirme Ricky: "Tous nos instruments, guitares, basse, batterie, ont été fabriqués de manière artisanale, par nous-mêmes. Nous y tenons beaucoup, parce que c'est avec ce son-là que nous avons gagné notre succès. Pour nous, c'est le nouveau son du monde. Si on prenait des guitares fabriquées par les Blancs, ça changerait notre son. Sur sa boîte de conserve et son petit fil de fer, Roger joue avec l'esprit !" On remarque également que s'il rassemble des foules européennes, le Staff reste un outsider pour le public congolais. Alors que la diaspora constitue la moitié voire les neuf dixièmes des spectateurs qui se pressent pour applaudir les stars congolaises internationales dont la musique est héritée de la rumba, elle ne forme qu'une petite minorité du public des concerts du Staff. Un phénomène récent accentue cette distance. Depuis plus d'un an, certains Congolais de la diaspora européenne regroupés sous le nom de "Combattants" ou "Bana Congo" s'évertuent à empêcher la tenue des concerts des vedettes congolaises qui ont chanté en faveur du pouvoir en place à Kinshasa. Ainsi, Werrason, Papa Wemba, Tshala Muana, Fally Ipupa et autres Koffi Olomide ne peuvent-ils quasiment plus se produire en Europe. Cette situation renforce non seulement le statut d'outsider du Staff Benda Bilili, mais aussi sa stature internationale. Alors que le groupe a toujours refusé jusqu'à la pratique, très répandue chez les artistes congolais, des dédicaces dans les chansons contre rémunération des personnes citées, il se trouve, par le jeu des circonstances, occuper actuellement la toute première place parmi les groupes kinois se produisant sur les scènes internationales. Depuis 2009, les tournées du Staff Benda Bilili ont conduit ses musiciens tout autour de la planète: Australie, Nouvelle-Zélande, Nouvelle-Calédonie, Guadeloupe, Martinique, États-Unis... Mais, paradoxalement, il est rare qu'il se produise à Kinshasa, sinon dans les lieux identifiés pour les Blancs : Halle de la Gombé, Centre Wallonie-Bruxelles ou dans les résidences de ambassadeurs. "Nos chansons passent à la radio, on nous voit à la télé, sur TV5 et les télévisions congolaises. Le public congolais commence maintenant à nous chercher à Kinshasa. Il veut savoir où l'on répète. Beaucoup de gens viennent à la maison pour écouter ce que nous faisons", témoigne Ricky. 


\section{Les chansons}

5 Grâce au succès remporté par le premier album, Très Très Fort, un deuxième, Bouger le Monde!, sort en septembre 2012. Ricky me propose d'en écouter un extrait. Il tire de sa poche son téléphone portable et cherche le morceau qu'il veut me faire entendre. Son visage s'éclaire d'un sourire quand se déclenche le son nasillard au rythme très entraînant de Mabele riche, où l'extraordinaire passion du chanteur animateur Kabamba Kabose Kasungo fait tout d'un coup monter l'ambiance à son climax. À écouter cette musique sur le minuscule haut-parleur d'un petit portable, je ne peux m'empêcher de repenser à ce temps pas si lointain (une quinzaine d'années), où le réseau téléphonique filaire du Zaïre était hors service. Seule une infime élite de possesseurs de téléphones satellitaires (au demeurant fort encombrants) étaient en mesure d'échanger des paroles avec leurs correspondants à l'intérieur ou à l'extérieur du pays... Ricky explique : "Partout là où nous sommes, nous faisons des chansons. J'écris quand vient l'inspiration. Nos paroles s'inspirent de ce qui nous entoure. Nous passons des messages. Dans Mabele riche, je dis que nous avons de très bonnes terres. 'Une terre riche !' Il suffit d'y jeter des graines pour voir les plantes pousser (...). Et lorsque nous mourons, notre corps vient enrichir la terre. Au Congo, nous avons beaucoup de minerais, qui pourraient faire notre richesse. Mais on ne sait pas comment les exploiter. Beaucoup d'Africains viennent en Europe pour étudier, mais quand ils y sont, ils se marient à la fin de leurs études. Ils restent en Europe et quand ils meurent, on ramène leur corps au Congo. Ça ne va pas! Dans ces conditions, comment allons-nous progresser en Afrique? Je dis que tous les jeunes qui viennent en Europe doivent ensuite rentrer au pays. Je m'adresse également aux enfants handicapés et à leurs familles. Chez nous, il faut faire vacciner les enfants, leur faire donner des soins par des kinés. Quand j'étais jeune, il n'y avait pas de vaccin, pas de kiné. C'est pour cela que nous sommes handicapés. Grâce aux succès du groupe, les musiciens ne circulent plus aujourd'hui sur leurs tricycles bricolés mais en voiture. Chacun a pu acquérir une parcelle où s'installer avec sa famille. Leurs enfants vont maintenant à l'école. Ricky n'a pas pour autant coupé ses relations avec le Centre pour handicapés dans lequel il vivait auparavant. Il y a formé un groupe de jeunes musiciens, auxquels il fournit équipement, conseils et encouragements. Une ONG, Benda Bilili, a été mise en place par les membres du groupe. Elle a pour objectif d'aider leurs anciens frères de misère à s'insérer dans le monde du travail. Un terrain a été acheté dans le but d'y bâtir une école pour les shégés et les handicapés. On leur enseigne la musique, la couture, la cordonnerie, l'ajustage et l'informatique. Et Ricky d'affirmer: "Mon rôle est de les encadrer, de les aider à trouver un travail pour qu'ils construisent leur vie." 


\section{Discographie}

- Très Très Fort, 2009

- Bouger le Monde !, 2012

\section{Vidéographie}

- Benda Bilili !, de Renaud Barret et Florent de La Tullaye, 2010

- Soul Power, de Jeffrey Levy-Hinte, 2009 